Bjorn Poonen

\title{
Existence of rational points on smooth projective varieties
}

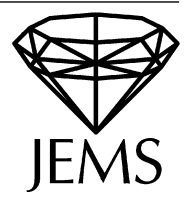

\author{
To Jean-Louis Colliot-Thélène on his $60^{\text {th }}$ birthday
}

Received March 20, 2007 and in revised form August 22, 2008

\begin{abstract}
Fix a number field $k$. We prove that if there is an algorithm for deciding whether a smooth projective geometrically integral $k$-variety has a $k$-point, then there is an algorithm for deciding whether an arbitrary $k$-variety has a $k$-point and also an algorithm for computing $X(k)$ for any $k$-variety $X$ for which $X(k)$ is finite. The proof involves the construction of a one-parameter algebraic family of Châtelet surfaces such that exactly one of the surfaces fails to have a $k$-point.
\end{abstract}

Keywords. Brauer-Manin obstruction, Hasse principle, Châtelet surface, conic bundle, rational points

\section{Statement of results}

Given a field $k$, a $k$-variety is a separated scheme of finite type over $k$. We will consider algorithms (Turing machines) accepting as input $k$-varieties where $k$ is a number field. Each such variety may be represented by a finite number of affine open patches together with gluing data, so it admits a finite description suitable for input into a Turing machine. We do not require algorithms to run in polynomial time or any other specified time, but they must terminate with an answer for each allowable input.

Theorem 1.1. Fix a number field $k$. Suppose that there exists an algorithm for deciding whether a regular projective geometrically integral $k$-variety has a $k$-point. Then

(i) there is an algorithm for deciding whether an arbitrary $k$-variety has a $k$-point,

(ii) there is an algorithm for computing $X(k)$ for any $k$-variety $X$ for which $X(k)$ is finite.

\section{Remark 1.2.}

(a) For a field $k$ of characteristic 0 , a $k$-variety is regular if and only if it is smooth over $k$. Nevertheless, we have two reasons for sometimes using the adjective "regular":

- In some situations, for instance when speaking of families of varieties, it helps to distinguish the absolute notion (regular) from the relative notion (smooth).

B. Poonen: Department of Mathematics, University of California, Berkeley, CA 94720-3840, USA; current address: Department of Mathematics, Massachusetts Institute of Technology, Cambridge, MA 02139-4307, USA; e-mail: poonen@ math.mit.edu; URL: http://math.mit.edu/ poonen/

Mathematics Subject Classification (2000): Primary 14G05; Secondary 11G35, 11U05, 14G25, $14 \mathrm{~J} 20$ 
- In Section 11, we say what can be said about the analogue for global function fields.

(b) For regular proper integral $k$-varieties, the property of having a $k$-point is a birational invariant, equivalent to the existence of a (not necessarily rank 1) valuation $v$ on the function field such that $v$ is trivial on $k$, and $k$ maps isomorphically to the residue field: this follows from [Nis55] and is also close to [Lan54, Theorem 3]; see also [CCS80, Lemme 3.1.1]. Thus one might wonder whether the decision problem is easier for regular projective geometrically integral varieties than for arbitrary ones. But Theorem 1.1(i) says that in fact the two problems are equivalent.

(c) For $k=\mathbb{Q}$, Theorem 1.1 (i) was more or less known: it is easily deduced from a result of R. Robinson [Smo91, §II.7] that the problem of deciding the existence of a rational zero of a polynomial over $\mathbb{Q}$ is equivalent to the problem of deciding the existence of a nontrivial rational zero of a homogeneous polynomial over $\mathbb{Q}$. Robinson's argument generalizes easily to number fields with a real place.

(d) Theorem 1.1 becomes virtually trivial if the word "projective" is changed to "affine". On the other hand, there are related statements for affine varieties that are nontrivial: for instance, if there is an algorithm for deciding whether any irreducible affine plane curve of geometric genus at least 2 has a rational point, then there is an algorithm for determining the set of rational points on any such curve [Kim03].

(e) By restriction of scalars, if we have an algorithm for deciding whether a regular projective geometrically integral $\mathbb{Q}$-variety has a rational point, then we have an analogous algorithm over any number field. But there is no number field for which the existence of such algorithms is known.

(f) Remark 8.2 will imply that to have algorithms as in (i) and (ii) of Theorem 1.1 for curves, it would suffice to be able to decide the existence of rational points on regular projective geometrically integral 3-folds. (If over $\mathbb{Q}$ one uses Robinson's reduction instead, one would need an algorithm for 9-folds!)

Theorem 1.1 will be deduced in Section 10 from the following:

Theorem 1.3. Let $k$ be a number field. Let $X$ be a projective $k$-variety. Let $U \subseteq X$ be an open subvariety. Then there exists a regular projective variety $Y$ and a morphism $\pi: Y \rightarrow X$ such that $\pi(Y(k))=U(k)$. Moreover, there exists an algorithm for constructing $(Y, \pi)$ given $(k, X, U)$.

The key special case, from which all others will be derived, is the case where $U=\mathbb{A}^{1}$ and $X=\mathbb{P}^{1}$. In this case we can arrange also for $\pi^{-1}(t)$ to be smooth and geometrically integral for all $t \in \mathbb{P}^{1}(k)$ : see Proposition 7.2 . Thus we will have a family of smooth projective geometrically integral varieties in which every rational fiber but one has a rational point, an extreme example of geometry not controlling arithmetic!

Remark 1.4. Theorem 1.3 fails for many fields $k$ that are not number fields, even for those that have a complicated arithmetic. Proposition 7.3 implies that it fails for the function field of any $\mathbb{C}$-variety, for instance. 


\section{Notation}

Let $k$ be a number field. Let $\mathcal{O}_{k}$ be the ring of integers in $k$. Let $\Omega_{k}$ be the set of places of $k$. If $v \in \Omega_{k}$, let $k_{v}$ be the completion of $k$ at $v$. If $v$ is nonarchimedean, let $\mathbb{F}_{v}$ be the residue field. Call $v$ odd if it is nonarchimedean and $\# \mathbb{F}_{v}$ is odd. If $a \in \mathcal{O}_{k}$ generates a prime ideal, let $v_{a}$ be the associated valuation, and let $\mathbb{F}_{a}=\mathbb{F}_{v_{a}}$. For $a \in k$, let $a \gg 0$ mean that $a$ is totally positive, i.e., positive for every real embedding of $k$. For any field $L$, let $L^{\times}$be the unit group $L-\{0\}$.

\section{Conic bundles}

A conic over $k$ is the zero locus in $\mathbb{P}^{2}=\operatorname{Proj} k\left[x_{0}, x_{1}, x_{2}\right]$ of a nonzero degree-2 homogeneous polynomial $s$ in $k\left[x_{0}, x_{1}, x_{2}\right]$. If $E$ is the $k$-vector space with basis $x_{0}, x_{1}, x_{2}$, then we may view $\mathbb{P}^{2}$ as $\mathbb{P} E:=\operatorname{Proj} \operatorname{Sym} E$, and $s$ as a nonzero element of $\operatorname{Sym}^{2} E$. By analogy, a conic bundle $C$ over a $k$-scheme $B$ is the zero locus in $\mathbb{P} \mathcal{E}$ of a nowherevanishing global section $s$ of $\operatorname{Sym}^{2} \mathcal{E}$, where $\mathcal{E}$ is some rank-3 vector sheaf on $B$. We will consider only the special case where $\mathcal{E}=\mathcal{L}_{0} \oplus \mathcal{L}_{1} \oplus \mathcal{L}_{2}$ for some line sheaves $\mathcal{L}_{i}$ and $s=s_{0}+s_{1}+s_{2}$ where $s_{i} \in \Gamma\left(B, \mathcal{L}_{i}^{\otimes 2}\right)$; we then call $C \rightarrow B$ a diagonal conic bundle.

Lemma 3.1. Let $B$ be a smooth curve over $k$. Let $\bar{k}$ be an algebraic closure of $k$. Let $C \rightarrow B$ be a diagonal conic bundle, with notation as above, such that $\sum_{i=0}^{2} \operatorname{ord}_{P}\left(s_{i}\right) \leq 1$ for every $P \in B(\bar{k})$. Then the total space $C$ is smooth over $k$.

Proof. We may assume that $k$ is algebraically closed. Let $\pi$ be the morphism $C \rightarrow B$. Since $B$ is smooth, $C$ is smooth over $k$ at any point where $\pi$ is smooth. Thus we need only check the singular points of the fibers of $\pi$.

Given $P \in B$, choose a neighborhood $U$ of $P$ in $B$ such that $\left.\mathcal{L}_{i}\right|_{U} \simeq \mathcal{O}_{U}$; then $\pi^{-1}(U)$ is isomorphic to a conic bundle $a_{0} x_{0}^{2}+a_{1} x_{1}^{2}+a_{2} x_{2}^{2}=0$ in $\mathbb{P}_{U}^{2}$ where the $a_{i} \in \mathcal{O}_{B}(U)$ satisfy $\sum_{i=0}^{2} \operatorname{ord}_{P}\left(a_{i}\right) \leq 1$. If all the $a_{i}$ are nonvanishing at $P$, then the fiber $\pi^{-1}(P)$ is a smooth conic. Otherwise, at most one of the $a_{i}$ vanishes at $P$, say ord $\operatorname{ord}_{P}\left(a_{2}\right)=$ 1. Then $\pi^{-1}(P)$ has a unique singularity $Q$, the point $((0,0), P)$ in the affine patch $a_{0} X_{0}^{2}+a_{1} X_{1}^{2}+a_{2}=0$ in the smooth $k$-variety $\mathbb{A}^{2} \times U$, where $X_{i}:=x_{i} / x_{2}$. Let $\mathfrak{m}_{Q}$ be the maximal ideal of $Q$ in $\mathbb{A}^{2} \times U$. Then $a_{0} X_{0}^{2}+a_{1} X_{1}^{2} \in \mathfrak{m}_{Q}^{2}$ but $\operatorname{ord}_{P}\left(a_{2}\right)=1$, so $a_{0} X_{0}^{2}+$ $a_{1} X_{1}^{2}+a_{2} \notin \mathfrak{m}_{Q}^{2}$. Thus $C$ is regular at $Q$, and hence $C$ is smooth over $k$ even at $Q$.

\section{Hilbert symbol}

For $v \in \Omega_{k}$ and $t, u \in k_{v}^{\times}$, let $(t, u)_{v} \in\{ \pm 1\}$ be the $v$-adic Hilbert symbol: by definition, $(t, u)_{v}=1$ if and only if $x^{2}-t y^{2}-u z^{2}=0$ has a solution $(x, y, z) \neq(0,0,0)$ in $k_{v}^{3}$. We recall some basic properties of the Hilbert symbol:

Lemma 4.1. For all $t, t^{\prime}, u, u^{\prime} \in k_{v}^{\times}$, we have

(a) $(t, u)_{v}=1$ if and only if $t$ belongs to the image of the norm map $k_{v}(\sqrt{u})^{\times} \rightarrow k_{v}^{\times}$.

(b) $(t, u)_{v}=(u, t)_{v}$.

(c) $\left(t t^{\prime}, u\right)_{v}=(t, u)_{v}\left(t^{\prime}, u\right)_{v}$ and $\left(t, u u^{\prime}\right)_{v}=(t, u)_{v}\left(t, u^{\prime}\right)_{v}$; in particular, $\left(t, u^{2}\right)_{v}=1$. 
(d) $(t,-t)_{v}=1$.

(e) $(t, 1-t)_{v}=1$, assuming in addition that $t \neq 1$.

(f) Suppose that $v$ is odd and $v(t)=0$. Then $(t, u)_{v}=-1$ if and only if $v(u)$ is odd and the image of $t$ in $\mathbb{F}_{v}$ is a nonsquare.

If $t, u \in k^{\times}$, we have the product formula

(g) $\prod_{v \in \Omega_{k}}(t, u)_{v}=1$.

Proof. See [Ser73, Chapter III] for the case $k=\mathbb{Q}$. See [Ser79, Chapter XIV] for the general case: in particular, Proposition 7 there yields (a)-(e); Proposition 8 implies (f); and p. 222 contains (g).

\section{Châtelet surfaces}

Fix $\alpha \in k^{\times}$and $P(x) \in k[x]$ of degree at most 4. Let $V_{0}$ be the affine surface in $\mathbb{A}^{3}$ given by $y^{2}-\alpha z^{2}=P(x)$. We want a smooth projective model $V$ of $V_{0}$. Define $\tilde{P}(w, x):=$ $w^{4} P(x / w)$; view $\tilde{P}$ as a section of $\mathcal{O}(4)$ on $\mathbb{P}^{1}:=\operatorname{Proj} k[w, x]$. The construction of Section 3 with $B=\mathbb{P}^{1}, \mathcal{L}_{0}=\mathcal{L}_{1}=\mathcal{O}, \mathcal{L}_{2}=\mathcal{O}(2), s_{0}:=1, s_{1}:=-\alpha$, and $s_{2}:=-\tilde{P}$ gives a diagonal conic bundle $V \rightarrow \mathbb{P}^{1}$ containing $V_{0}$ as an affine open subvariety. Since $V \rightarrow \mathbb{P}^{1}$ is projective, $V$ is projective over $k$ too. If $P(x)$ is not identically 0 , then $V$ is geometrically integral. If $P(x)$ is separable and of degree 3 or 4 , then $\tilde{P}(w, x)$ is separable and $V$ is smooth over $k$ by Lemma 3.1 , in this case $V$ is called the Chatelet surface given by $y^{2}-\alpha z^{2}=P(x)$.

Iskovskikh [Isk71] showed that the Châtelet surface over $\mathbb{Q}$ given by

$$
y^{2}+z^{2}=\left(x^{2}-2\right)\left(3-x^{2}\right)
$$

violated the Hasse principle. Several years later it was shown that this violation could be explained by the Brauer-Manin obstruction, and that more generally, any Châtelet surface over a number field given by $y^{2}-a z^{2}=f(x) g(x)$ with $f$ and $g$ distinct irreducible quadratic polynomials satisfies the Hasse principle if and only if there is no BrauerManin obstruction [CCS80, Theorem B]. Finally, the two-part paper [CSS87a, CSS87b] generalized this to all Châtelet surfaces over number fields. For an introduction to the Brauer-Manin obstruction, see [Sko01, §5.2].

Proposition 5.1. There exists a Châtelet surface $V$ over $k$ that violates the Hasse principle.

The rest of this section is devoted to the proof of Proposition 5.1, so a reader interested in only the case $k=\mathbb{Q}$ may accept the Iskovskikh example and proceed to Section 6 We generalize the argument presented in [Sko01, p. 145].

By the Chebotarev density theorem and global class field theory applied to a ray class field, we can find $b \in \mathcal{O}_{k}$ generating a prime ideal such that $b \gg 0$ and $b \equiv 1\left(\bmod 8 \mathcal{O}_{k}\right)$. Similarly we find $a \in \mathcal{O}_{k}$ generating a prime ideal such that $a \gg 0$ and $a \equiv 1\left(\bmod 8 \mathcal{O}_{k}\right)$ and $a$ is a not a square modulo $b$. We may assume that $\# \mathbb{F}_{a}, \# \mathbb{F}_{b}>5$. Fix $c \in \mathcal{O}_{k}$ such that $b \mid(a c+1)$.

We use the abbreviation $(t, u)_{b}:=(t, u)_{v_{b}}$. We will need the following Hilbert symbol calculations later: 
Lemma 5.2. We have

(i) $(-1, a)_{v}=1$ for all $v \in \Omega_{k}$.

(ii) $(-1, b)_{v}=1$ for all $v \in \Omega_{k}$.

(iii) $(a b, a)_{b}=-1$.

(iv) $(a b, c)_{b}=-1$.

Proof.

(i) For $v$ archimedean or 2-adic, we have $a \in k_{v}^{\times 2}$, so Lemma 4.1 (c) implies $(-1, a)_{v}$ $=1$. For all other $v$ except $v_{a}$, we have $v(-1)=v(a)=0$, so Lemma 4.1(f) implies $(-1, a)_{v}=1$. For $v=v_{a}$, it follows from Lemma 4.1 $\left.\mathrm{g}\right)$.

(ii) The proof is the same as that of (i).

(iii) By (i) and Lemma 4.1 c, d,f), we have $(a b, a)_{b}=(-1, a)_{b}(a b, a)_{b}=$ $(-a, a)_{b}(b, a)_{b}=1 \cdot(b, a)_{b}=-1$.

(iv) Since $b \mid(a c+1)$, we have $a c \in(-1) k_{v_{b}}^{\times 2}$, so Lemma $4.1(\mathrm{c})$ implies $(a b, a c)_{b}=$ $(a b,-1)_{b}=(a,-1)_{b}(b,-1)_{b}=1$, where we used (i) and (ii) in the last step. Divide by (iii) to get $(a b, c)_{b}=-1$.

Let $V$ be the Châtelet surface given by

$$
y^{2}-a b z^{2}=\left(x^{2}+c\right)\left(a x^{2}+a c+1\right) .
$$

(The quadratic factors on the right are separable and generate the unit ideal of $k[x]$, so $V$ is smooth over $k$.)

Lemma 5.3. The variety $V$ has a $k_{v}$-point for every place $v$ of $k$.

Proof. Suppose that $v$ is archimedean or 2-adic. Then $a b \in k_{v}^{\times 2}$, so the left hand side of (1) factors as $(y+d z)(y-d z)$ for some $d \in k_{v}^{\times}$; now, choose $x \in k_{v}$, write the value of the right hand side of (1) as $x_{1} x_{2}$ for some $x_{1}, x_{2} \in k_{v}$, and solve the system $y+d z=x_{1}$, $y-d z=x_{2}$ for $y, z \in k_{v}$ to obtain a $k_{v}$-point of $V$.

Suppose that $v$ is odd and $v \notin\left\{v_{a}, v_{b}\right\}$. Choose $x \in k$ with $v(x)<0$. Then the right hand side of (1) has even valuation and is hence a norm for the unramified extension $k_{v}(\sqrt{a b}) / k_{v}$. So $V$ has a $k_{v}$-point.

Suppose that $v=v_{b}$. Because $a$ is not a square modulo $b$, all $\mathbb{F}_{b}$-points on the projective closure of the affine curve $y^{2}=a\left(x^{2}+c\right)$ over $\mathbb{F}_{b}$ lie on the affine part, so there are $\# \mathbb{F}_{b}+1$ solutions $(x, y) \in \mathbb{F}_{b}^{2}$. Then the number of solutions with $x^{2}+c \neq 0$ and $x \neq 0$ is at least $\left(\# \mathbb{F}_{b}+1\right)-2-2-2>0$. Choose $x \in \mathcal{O}_{k}$ reducing to the $x$-coordinate of such a solution. The right hand side of (1) is congruent modulo $b$ to $\left(x^{2}+c\right)\left(a x^{2}\right)$, so by Hensel's lemma it is in $k_{v}^{\times 2}$. Thus $V$ has a $k_{v}$-point.

Suppose that $v=v_{a}$. The same argument as in the previous paragraph shows that we may choose $x \in \mathcal{O}_{k}$ such that $x^{2}+c \in k_{v}^{\times 2}$. The other factor $a x^{2}+a c+1$ is $1 \bmod a$, hence in $k_{v}^{\times 2}$. Therefore the right hand side of (1) is in $k_{v}^{\times 2}$, so $V$ has a $k_{v}$-point.

Let $\kappa(V)$ be the function field of $V$. Let $A \in \operatorname{Br} \kappa(V)$ be the class of the quaternion algebra $\left(a b, x^{2}+c\right)$. Since for any $g \in \kappa(V)^{\times}$the class of $(a b, g)$ is unaffected by multiplying $g$ by a square or by a norm from $\kappa(V)(\sqrt{a b})$, the class $A$ equals the class of $\left(a b, 1+c / x^{2}\right)$ and of $\left(a b, a x^{2}+a c+1\right)$. 
Lemma 5.4. The element $A$ belongs to the subgroup $\operatorname{Br} V$ of $\operatorname{Br} \kappa(V)$.

Proof. First of all, $V$ is a regular integral scheme, so $\operatorname{Br} V$ is a subgroup of $\operatorname{Br} \kappa(V)$, and it consists of the elements whose residue at every codimension-1 point $P$ of $V$ vanishes. To check that $A$ satisfies this residue condition at $P$, it is sufficient to show that $A$ can be represented by a quaternion algebra $(f, g)$ where $f, g \in \kappa(V)^{\times}$are regular and nonvanishing at $P$. In fact, at every $P \in V$, one of the three representations of $A$ given in the paragraph preceding Lemma 5.4 is of this form.

We will show that $A$ gives a Brauer-Manin obstruction to the Hasse principle. For $P_{v} \in$ $V\left(k_{v}\right)$, let $A\left(P_{v}\right) \in \mathrm{Br} k_{v}$ be the evaluation of $A$ at $P_{v}$. Let $\operatorname{inv}_{v}: \operatorname{Br} k_{v} \hookrightarrow \mathbb{Q} / \mathbb{Z}$ be the usual invariant map. Given $P_{v} \in V\left(k_{v}\right)$, if $A$ is represented by $(f, g)$ with $f, g \in \kappa(V)^{\times}$ regular and nonvanishing at $P_{v}$, then $\operatorname{inv}_{v}\left(A\left(P_{v}\right)\right)$ is 0 or $1 / 2$ according to whether the Hilbert symbol $\left(f\left(P_{v}\right), g\left(P_{v}\right)\right)_{v}$ is 1 or -1 .

Lemma 5.5. For any $P_{v} \in V\left(k_{v}\right)$,

$$
\operatorname{inv}_{v}\left(A\left(P_{v}\right)\right)= \begin{cases}0 & \text { if } v \neq v_{b}, \\ 1 / 2 & \text { if } v=v_{b} .\end{cases}
$$

Proof. Since $V$ is smooth, the implicit function theorem shows that $V_{0}\left(k_{v}\right)$ is $v$-adically dense in $V\left(k_{v}\right)$. Since $\operatorname{inv}_{v}\left(A\left(P_{v}\right)\right)$ is a continuous function on $V\left(k_{v}\right)$ with the $v$-adic topology, it suffices to prove the result for $P_{v} \in V_{0}\left(k_{v}\right)$.

Suppose that $v$ is archimedean or 2-adic. Then $a b \in k_{v}^{\times 2}$, so for any $t \in k_{v}^{\times}$the Hilbert symbol $(a b, t)_{v}$ is 1 . Hence $\operatorname{inv}_{v}\left(A\left(P_{v}\right)\right)=0$.

Suppose that $v$ is odd and $v \notin\left\{v_{a}, v_{b}\right\}$. If $v(x)<0$ at $P_{v}$, then $v\left(x^{2}+c\right)$ is even, so Lemma 4.1 (f) implies $\operatorname{inv}_{v}\left(A\left(P_{v}\right)\right)=0$. If $v(x) \geq 0$, then either $x^{2}+c$ or $a x^{2}+a c+1$ is a $v$-adic unit, so using an appropriate representation of $A$ and applying Lemma 4.1 ff) shows that $\operatorname{inv}_{v}\left(A\left(P_{v}\right)\right)=0$.

Suppose that $v=v_{a}$. If $v(x)<0$ at $P_{v}$, then $x^{2}+c \in k_{v}^{\times 2}$, so Lemma 4.1 (c) implies $\operatorname{inv}_{v}\left(A\left(P_{v}\right)\right)=0$. If $v(x) \geq 0$, then $a x^{2}+a c+1$ is $1 \bmod a$ so it is in $k_{v}^{\times 2}$, and again $\operatorname{inv}_{v}\left(A\left(P_{v}\right)\right)=0$.

Finally, suppose that $v=v_{b}$. Each of the following two sentences will use the following observation: if elements $t, u \in k_{v}^{\times}$and $\epsilon \in k_{v}$ satisfy $v(u) \leq 0<v(\epsilon)$, then $(u+\epsilon) / u \in k_{v}^{\times 2}$, so Lemma 4.1.c) implies $(t, u+\epsilon)_{b}=(t, u)_{b}$. If $v(x) \leq 0$, then taking $\epsilon=a c+1$ yields $\left(a b, a x^{2}+a c+1\right)_{b}=\left(a b, a x^{2}\right)_{b}=(a b, a)_{b}=-1$, by Lemma 5.2 (iii). If $v(x)>0$, then taking $\epsilon=x^{2}$ yields $\left(a b, x^{2}+c\right)_{b}=(a b, c)_{b}=-1$, by Lemma 5.2(iv). In either case, $\operatorname{inv}_{v}\left(A\left(P_{v}\right)\right)=1 / 2$.

Lemma 5.5, together with the reciprocity law $\sum_{v \in \Omega_{k}} \operatorname{inv}_{v}(\alpha)=0$ for $\alpha \in \operatorname{Br} k$ (or the special case for quaternion algebras given by Lemma 4.1 (g)), implies that $V$ has no $k$ point. This completes the proof of Proposition 5.1 .

\section{Châtelet surface bundles}

By a Châtelet surface bundle over $\mathbb{P}^{1}$ we mean a flat proper morphism $\mathcal{V} \rightarrow \mathbb{P}^{1}$ such that the generic fiber is a Châtelet surface; then for $t \in \mathbb{P}^{1}(k)$, we let $\mathcal{V}_{t}$ be the fiber above $t$. 
We retain the notation of Section 5 . Let $\tilde{P}_{0}(w, x) \in k[w, x]$ be the homogeneous form of degree 4 obtained by homogenizing the right hand side of $(1)$. Let $\tilde{P}_{\infty}(w, x)$ be any irreducible degree-4 form in $k[w, x]$. Thus $\tilde{P}_{0}$ and $\tilde{P}_{\infty}$ are linearly independent.

Let $\mathcal{V}$ be the diagonal conic bundle over $\mathbb{P}^{1} \times \mathbb{P}^{1}:=\operatorname{Proj} k[u, v] \times \operatorname{Proj} k[w, x]$ obtained by taking $\mathcal{L}_{0}=\mathcal{L}_{1}:=\mathcal{O}, \mathcal{L}_{2}:=\mathcal{O}(1,2), s_{0}:=1, s_{1}:=-a b$, and $s_{2}:=$ $-\left(u^{2} \tilde{P}_{\infty}+v^{2} \tilde{P}_{0}\right)$. Composing $\mathcal{V} \rightarrow \mathbb{P}^{1} \times \mathbb{P}^{1}$ with the first projection $\mathbb{P}^{1} \times \mathbb{P}^{1} \rightarrow \mathbb{P}^{1}$ lets us view $\mathcal{V}$ as a Châtelet surface bundle over $\mathbb{P}^{1}=\operatorname{Proj} k[u, v]$ with projective geometrically integral fibers. If $u, v \in k$ are not both 0 , the fiber above $(u: v) \in \mathbb{P}^{1}(k)$ is the Châtelet surface given by

$$
y^{2}-a b z^{2}=u^{2} \tilde{P}_{\infty}(1, x)+v^{2} \tilde{P}_{0}(1, x),
$$

if smooth over $k$. In particular, the fiber $\mathcal{V}_{(0: 1)}$ is isomorphic to $V$.

Call a subset $T$ of $\mathbb{P}^{1}(k)$ thin if and only if there exist finitely many regular projective geometrically integral curves $C_{i}$ and morphisms $v_{i}: C_{i} \rightarrow \mathbb{P}^{1}$ of degree greater than 1 such that $T \subseteq \bigcup v_{i}\left(C_{i}(k)\right)$; cf. [Ser97, §9.1]. Such sets arise in the context of the Hilbert irreducibility theorem.

Lemma 6.1. The set of specializations $(u: v) \in \mathbb{P}^{1}(k)$ such that $u^{2} \tilde{P}_{\infty}+v^{2} \tilde{P}_{0} \in k[w, x]$ is reducible (for any or all choices of $(u, v) \in k^{2}-\{(0,0)\}$ representing $(u: v)$ ) is a thin set.

Proof. We may assume $u=1$. The degree- 4 form $\tilde{P}_{\infty}+v^{2} \tilde{P}_{0}$ over $k(v)$ is irreducible since it has an irreducible specialization, namely $\tilde{P}_{\infty}$. Apply [Ser97, §9.2, Proposition 1].

Lemma 6.2. There exists a finite set $S$ of noncomplex places of $k$ and a neighborhood $N_{v}$ of $(0: 1)$ in $\mathbb{P}^{1}\left(k_{v}\right)$ for each $v \in S$ such that for $t \in \mathbb{P}^{1}(k)$ belonging to $N_{v}$ for each $v \in S$, the fiber $\mathcal{V}_{t}$ has a $k_{v}$-point for every $v \in \Omega_{k}$.

Proof. This is an application of the "fibration method", which has been used previously in various places (e.g., [CSS87a], [Col98, 2.1], [CP00, Lemma 3.1]). Since all geometric fibers of the $k$-morphism $\mathcal{V} \rightarrow \mathbb{P}^{1}$ are integral, the same is true for a model over some ring $\mathcal{O}_{k, S}$ of $S$-integers. By adding finitely many $v$ to $S$, we can arrange that for nonarchimedean $v \notin S$ the residue field $\mathbb{F}_{v}$ is large enough that every $\mathbb{F}_{v}$-fiber has a smooth $\mathbb{F}_{v}$-point by the Weil conjectures; then by Hensel's lemma any $k_{v}$-fiber has a $k_{v}$-point. Include the real places in $S$, and exclude the complex places since for complex $v$ the existence of $k_{v}$-points on fibers is automatic. For $v \in S$, since the fiber above $(0: 1)$ has a $k_{v}$-point, and since $\mathcal{V} \rightarrow \mathbb{P}^{1}$ is smooth above $(0: 1)$, the implicit function theorem implies that the image of $\mathcal{V}\left(k_{v}\right) \rightarrow \mathbb{P}^{1}\left(k_{v}\right)$ contains a $v$-adic neighborhood $N_{v}$ of $(0: 1)$ in $\mathbb{P}^{1}\left(k_{v}\right)$.

\section{Base change}

The following lemma combines the idea of [CP00, Lemma 3.3] with some new ideas.

Lemma 7.1. Let $P \in \mathbb{P}^{1}(k)$. Let $S$ be a finite set of noncomplex places of $k$. For each $v \in S$, let $N_{v}$ be a neighborhood of $P$ in $\mathbb{P}^{1}\left(k_{v}\right)$. Let $T$ be a thin subset of $\mathbb{P}^{1}(k)$ con- 
taining $P$. Then there exists a $k$-morphism $\gamma: \mathbb{P}^{1} \rightarrow \mathbb{P}^{1}$ such that both of the following hold:

(1) $\gamma\left(\mathbb{P}^{1}\left(k_{v}\right)\right) \subseteq N_{v}$ for each $v \in S$.

(2) $\gamma^{-1}(T) \cap \mathbb{P}^{1}(k)$ consists of a single point $Q$ with $\gamma(Q)=P$.

Proof. We will construct $\gamma$ as a composition. But we present the argument as a series of reductions, each step of which involves taking the inverse image of all the data under some $\beta: \mathbb{P}^{1} \rightarrow \mathbb{P}^{1}$ and replacing $P$ by some $P^{\prime} \in \beta^{-1}(P) \cap \mathbb{P}^{1}(k)$.

Choose finitely many regular projective geometrically integral curves $C_{i}$ and morphisms $v_{i}: C_{i} \rightarrow \mathbb{P}^{1}$ of degree greater than 1 such that $T \subseteq \bigcup v_{i}\left(C_{i}(k)\right)$. By choosing a suitable coordinate on $\mathbb{P}^{1}$, we may assume that $0, \infty \in \mathbb{P}^{1}(k)$ are disjoint from the branch points of every $v_{i}$, and that $1 \in \mathbb{P}^{1}(k)$ is the point $P$. Choose $n \in \mathbb{Z}_{>0}$ such that $n>2 \operatorname{deg} v_{i}$ for every $i$, and let $\beta: \mathbb{P}^{1} \rightarrow \mathbb{P}^{1}$ be the morphism corresponding to the rational function $t \mapsto t^{n}$; note that there exists $P^{\prime} \in \mathbb{P}^{1}(k)$ with $\beta\left(P^{\prime}\right)=P$. Define the fiber product $C_{i}^{\prime}$ with morphisms $v_{i}^{\prime}: C_{i}^{\prime} \rightarrow \mathbb{P}^{1}$ and $\beta_{i}: C_{i}^{\prime} \rightarrow C_{i}$ making a cartesian diagram

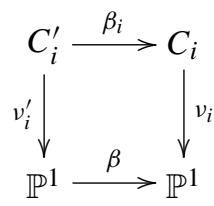

Since $\beta$ is totally ramified above 0 , the morphism $\beta_{i}$ is totally ramified above $v_{i}^{-1}(0)$, so $C_{i}^{\prime}$ is geometrically integral. Since the branch loci of $\beta$ and $v_{i}$ are disjoint, $C_{i}^{\prime}$ is regular and the ramification divisors $R_{i}^{\prime}$ and $R_{i}$ of $v_{i}^{\prime}$ and $v_{i}$, respectively, satisfy $R_{i}^{\prime}=\beta_{i}^{*} R_{i}$. Since $\mathbb{P}^{1}$ has no everywhere unramified cover, deg $R_{i}>0$. By the Hurwitz formula, the genus $g_{i}^{\prime}$ of $C_{i}^{\prime}$ satisfies

$$
2 g_{i}^{\prime}-2=\left(\operatorname{deg} v_{i}^{\prime}\right)(-2)+\operatorname{deg} R_{i}^{\prime}=\left(\operatorname{deg} v_{i}\right)(-2)+n \operatorname{deg} R_{i} \geq-2 \operatorname{deg} v_{i}+n>0,
$$

so $g_{i}^{\prime}>1$. By Faltings' theorem [Fal83], $C_{i}^{\prime}(k)$ is finite. We have $\beta^{-1}(T) \cap \mathbb{P}^{1}(k) \subseteq$ $\bigcup v_{i}^{\prime}\left(C_{i}^{\prime}(k)\right)$, so $\beta^{-1}(T) \cap \mathbb{P}^{1}(k)$ is finite. By pulling all the data back under $\beta$ and replacing $P$ by $P^{\prime}$, we reduce to the case where $T$ is finite.

Choose a new coordinate on $\mathbb{P}^{1}$ for which $P$ is $0 \in \mathbb{P}^{1}(k)$. Then the rational function $t \mapsto 1 /\left(t^{2}+m\right)$ maps $\infty$ to 0 and maps $\mathbb{P}^{1}(\mathbb{R})$ into $N_{v}$ for each real $v$ if $m \in \mathbb{Z}_{>0}$ is chosen large enough. Pulling all the data back under the corresponding endomorphism of $\mathbb{P}^{1}$, we reduce to the case where $S$ contains no archimedean places. Now suppose that $S$ contains a nonarchimedean place $v$. Let $q=\# \mathbb{F}_{v}$. Choose a large positive integer $r$, and let $m:=$ $q^{r}(q-1)$. Then the rational function $t \mapsto t^{m}$ maps all $t \in k_{v}$ with $v(t)>0$ into a small $v$ adic neighborhood of 0 , all $t \in k_{v}$ with $v(t)<0$ (including $\infty$ ) into a small neighborhood of $\infty$, and all $t \in k_{v}$ with $v(t)=0$ into a small neighborhood of 1 (raising to the power $q-1$ already maps the $t$ with $v(t)=0$ into the 1-units, and successively raising to the power $q$ brings these closer and closer to 1). Choose a rational function $g$ mapping $\{0,1, \infty\}$ to $P$; then choosing $r$ large enough arranges that the rational function $g\left(t^{m}\right)$ 
maps $\mathbb{P}^{1}\left(k_{v}\right)$ into $N_{v}$. Pulling back everything under the corresponding endomorphism of $\mathbb{P}^{1}$ lets us replace $S$ by $S-\{v\}$. Eventually we reduce to the case in which $S=\emptyset$.

For a suitable choice of coordinate, $P$ is the point $0 \in \mathbb{P}^{1}(k)$, and $\infty \notin T$. Choose $c \in k^{\times}$such that the images of $c$ and $T-\{0\}$ in $k^{\times} / k^{\times 2}$ do not meet. Let $\gamma: \mathbb{P}^{1} \rightarrow \mathbb{P}^{1}$ be given by the rational function $c t^{2}$. Then $\gamma^{-1}(T) \cap \mathbb{P}^{1}(k)$ consists of the single point 0 .

Proposition 7.2. There exists a Châtelet surface bundle $\mu: \mathcal{W} \rightarrow \mathbb{P}^{1}$ over $k$ such that

(i) $\mu$ is smooth over $\mathbb{P}^{1}(k)$,

(ii) $\mu(\mathcal{W}(k))=\mathbb{A}^{1}(k)$.

Proof. Obtain $\gamma: \mathbb{P}^{1} \rightarrow \mathbb{P}^{1}$ from Lemma 7.1 with $P=(0: 1)$, with $S$ and $N_{v}$ as in Lemma 6.2 and with $T$ the thin set of Lemma 6.1, note that $T$ contains the finitely many $t \in \mathbb{P}^{1}(k)$ above which $\mathcal{V} \rightarrow \mathbb{P}^{1}$ is not smooth. We may assume that the $Q$ in Lemma 7.1 is $\infty$. Define $\mathcal{W}$ as the fiber product

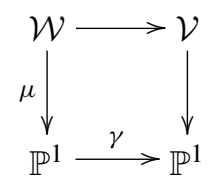

and let $\mu$ be the projection $\mathcal{W} \rightarrow \mathbb{P}^{1}$ shown. Then $\mu$ is smooth above $\mathbb{P}^{1}(k)$, and for every $t \in \mathbb{P}^{1}(k)$ the fiber $\mathcal{W}_{t}$ has a $k_{v}$-point for every $v$. If $t \in \mathbb{A}^{1}(k)$, then $\gamma(t) \notin T$, hence $\mathcal{W}_{t}$ is a Châtelet surface defined by an irreducible degree-4 polynomial, so by [CSS87a, Theorem B(i)(b)], $\mathcal{W}_{t}$ satisfies the Hasse principle; thus $\mathcal{W}_{t}$ has a $k$-point. But if $t=\infty$, then $\mathcal{W}_{t}$ is isomorphic to $\mathcal{V}_{(0: 1)} \simeq V$, which has no $k$-point.

The following proposition will not be needed elsewhere. Its role is only to illustrate that Theorem 1.3 and Proposition 7.2 depend subtly upon properties of $k$ : for instance, they are not true over all fields of cohomological dimension 2.

Proposition 7.3. Let $k_{0}$ be an uncountable algebraically closed field, and let $k$ be a field extension of $k_{0}$ generated by a set $S$ of cardinality less than $\# k_{0}$. Then there is no morphism $\pi: \mathcal{W} \rightarrow \mathbb{P}^{1}$ of projective $k$-varieties such that $\pi(\mathcal{W}(k))=\mathbb{A}^{1}(k)$.

Proof. Suppose that $\pi(\mathcal{W}(k))=\mathbb{A}^{1}(k)$. Fix a projective embedding $\mathcal{W} \hookrightarrow \mathbb{P}^{n}$. Let $\Gamma \subseteq \mathcal{W} \times \mathbb{P}^{1} \subseteq \mathbb{P}^{n} \times \mathbb{P}^{1}$ be the graph of $\pi$. Since $\mathcal{W}$ is projective, $\Gamma$ is the zero locus of a finite set of bihomogeneous polynomials $\gamma_{i}$ with coefficients in $k$.

Let $L$ be a finite-dimensional $k_{0}$-subspace of $k$. Let

$$
\mathbb{P}^{n}[L]=\left\{\left(a_{0}: \cdots: a_{n}\right) \in \mathbb{P}^{n}(k) \mid\left(a_{0}, \ldots, a_{n}\right) \in L^{n}-\{0\}\right\} .
$$

Let $\mathcal{W}[L]=\mathbb{P}^{n}[L] \cap \mathcal{W}(k) \subseteq \mathbb{P}^{n}(k)$.

We claim that the subset $I_{L}:=\pi(\mathcal{W}[L]) \cap \mathbb{P}^{1}\left(k_{0}\right)$ of $\mathbb{P}^{1}(k)$ is finite. Choose a $k_{0^{-}}$ basis of $L$ to identify $\left(L^{n+1}-\{0\}\right) / k_{0}^{\times}$with $\mathbb{P}^{N}\left(k_{0}\right)$, where $N+1=(n+1) \operatorname{dim}_{k_{0}} L$. For each $i$, the coefficients obtained when the value of $\gamma_{i}$ at

$$
\left(v_{0}, \ldots, v_{N}, w_{0}, w_{1}\right) \in k_{0}^{N+1} \times k_{0}^{2} \simeq L^{n+1} \times k_{0}^{2}
$$


is expressed as a linear combination of elements in a fixed $k_{0}$-basis of $k$ are bihomogeneous polynomials in $k_{0}\left[v_{0}, \ldots, v_{N} ; w_{0}, w_{1}\right]$. These bihomogeneous polynomials, taken for all $i$, define a Zariski closed subset $C_{L} \subseteq \mathbb{P}^{N}\left(k_{0}\right) \times \mathbb{P}^{1}\left(k_{0}\right)$. By definition of $\Gamma$ and the $\gamma_{i}$, the projection of $C_{L}$ onto the second factor equals $I_{L}$. Thus $I_{L}$ is Zariski closed in $\mathbb{P}^{1}\left(k_{0}\right)$. On the other hand, $I_{L} \subseteq \pi(\mathcal{W}[L]) \subseteq \pi(\mathcal{W}(k))=\mathbb{A}^{1}(k)$, so $\infty \notin I_{L}$. Thus $I_{L}$ is finite, as claimed.

Let $\mathcal{L}$ be the collection of finite-dimensional $k_{0}$-subspaces $L$ of $k$ spanned by a finite set of monomials in the elements of $S$. Then $\bigcup_{L \in \mathcal{L}} L$ is the $k_{0}$-subalgebra of $k$ generated by $S$, and its fraction field is $k$. Therefore $\bigcup_{L \in \mathcal{L}} \mathbb{P}^{n}[L]=\mathbb{P}^{n}(k)$ and $\bigcup_{L \in \mathcal{L}} \mathcal{W}[L]=$ $\mathcal{W}(k)$. Applying $\pi$ and intersecting with $\mathbb{P}^{1}\left(k_{0}\right)$ yields $\bigcup_{L \in \mathcal{L}} I_{L}=\pi(\mathcal{W}(k)) \cap \mathbb{P}^{1}\left(k_{0}\right)$. Thus

$$
\#\left(\pi(\mathcal{W}(k)) \cap \mathbb{P}^{1}\left(k_{0}\right)\right)=\# \bigcup_{L \in \mathcal{L}} I_{L} \leq \# \mathcal{L} \cdot \aleph_{0}=\max \left\{\# S, \aleph_{0}\right\}<\# k_{0}=\# \mathbb{A}^{1}\left(k_{0}\right) .
$$

The strict inequality implies $\pi(\mathcal{W}(k)) \neq \mathbb{A}^{1}(k)$.

\section{Reductions}

Lemma 8.1. There exists a projective $k$-variety $Z$ and a morphism $\eta: Z \rightarrow \mathbb{P}^{n}$ such that $\eta(Z(k))=\mathbb{A}^{n}(k)$ and $\eta$ is smooth above $\mathbb{A}^{n}(k)$.

Proof. Start with the birational map $\left(\mathbb{P}^{1}\right)^{n}-\rightarrow \mathbb{P}^{n}$ given by the isomorphism $\left(\mathbb{A}^{1}\right)^{n} \rightarrow$ $\mathbb{A}^{n}$. Resolve the indeterminacy, i.e., find a projective $k$-variety $J$ and a birational morphism $J \rightarrow\left(\mathbb{P}^{1}\right)^{n}$ whose composition with $\left(\mathbb{P}^{1}\right)^{n} \rightarrow \mathbb{P}^{n}$ extends to a morphism $J \rightarrow \mathbb{P}^{n}$ that is an isomorphism above $\mathbb{A}^{n}$. Define $Z$ to make a cartesian square

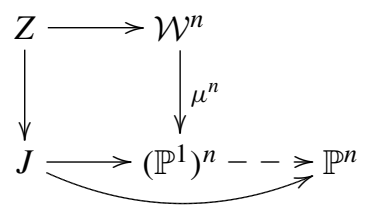

where $\mathcal{W} \stackrel{\mu}{\rightarrow} \mathbb{P}^{1}$ is as in Proposition 7.2 Let $\eta$ be the composition $Z \rightarrow J \rightarrow \mathbb{P}^{n}$.

By construction of $\mathcal{W}$, we have $\mu^{n}\left(\mathcal{W}^{n}(k)\right)=\left(\mathbb{A}^{1}\right)^{n}(k)$, so the image of $Z(k) \rightarrow$ $J(k)$ is contained in the copy of $\mathbb{A}^{n}$ in $J$. Therefore $\eta(Z(k)) \subseteq \mathbb{A}^{n}(k)$.

On the other hand, if $t \in \mathbb{A}^{n}(k)$, then $J \rightarrow\left(\mathbb{P}^{1}\right)^{n}$ is a local isomorphism above $t$, and $\mathcal{W}^{n} \rightarrow\left(\mathbb{P}^{1}\right)^{n}$ is smooth above $t$, so $Z \rightarrow J$ is smooth above $t$, and the fiber $\eta^{-1}(t)$ is isomorphic to the corresponding fiber of $\mathcal{W}^{n} \rightarrow\left(\mathbb{P}^{1}\right)^{n}$ so it has a $k$-point. Thus $\eta(Z(k))=\mathbb{A}^{n}(k)$.

Proof of existence in Theorem 1.3 . We use strong induction on $\operatorname{dim} X$. The case where $X$ is empty is trivial. We may assume that $X$ is integral; then $X$ is generically smooth, and the nonsmooth locus $X_{\text {sing }}$ is of lower dimension. Let $U_{\text {sing }}=U \cap X_{\text {sing. The inductive }}$ hypothesis gives $\pi_{1}: Y_{1} \rightarrow X_{\text {sing }}$ such that $\pi_{1}\left(Y_{1}(k)\right)=U_{\text {sing }}(k)$. If we prove the conclusion for the smooth open subvariety $U-U_{\text {sing }} \subseteq X$, i.e., if we find $\pi_{2}: Y_{2} \rightarrow X$ such 
that $\pi_{2}\left(Y_{2}(k)\right)=\left(U-U_{\text {sing }}\right)(k)$, then the disjoint union $Y_{1} U Y_{2}$ serves as a $Y$ for $U \subseteq X$. Thus we reduce to the case where $U$ is smooth over $k$.

If $U$ is a finite union of open subvarieties $U_{i}$, then it suffices to prove the conclusion for each $U_{i} \subseteq X$ and take the disjoint union of the resulting $Y$ 's. In particular, by choosing a projective embedding of $X$ and expressing $X-U$ as a finite intersection of hypersurface sections of $X$, we may reduce to the case where $U=X-D$ for some very ample effective divisor $D \subseteq X$. In other words, we may assume that $X \subseteq \mathbb{P}^{n}$ and $U=X \cap \mathbb{A}^{n}$.

Let $Z \rightarrow \mathbb{P}^{n}$ be as in Lemma 8.1. Define $Y_{0}$ to make a cartesian diagram

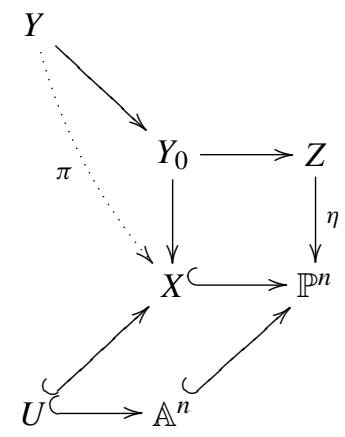

and let $Y \rightarrow Y_{0}$ be a resolution of singularities that is an isomorphism above the smooth locus of $Y_{0}$, so $Y$ is a regular projective variety. Let $\pi$ be the composition $Y \rightarrow Y_{0} \rightarrow X$.

Suppose that $t \in U(k)$. Then $Z \rightarrow \mathbb{P}^{n}$ is smooth above $t$, by choice of $Z$. So $Y_{0} \rightarrow X$ is smooth above $t$. Moreover, $U \rightarrow$ Spec $k$ is smooth, so $Y_{0} \rightarrow \operatorname{Spec} k$ is smooth above $t$. Therefore $Y \rightarrow Y_{0}$ is a local isomorphism above $t$. Thus $\pi^{-1}(t) \simeq \eta^{-1}(t)$, and the latter has a $k$-point.

On the other hand, if $t \in X(k)-U(k)$, then $\pi^{-1}(t)$ cannot have a $k$-point, since such a $k$-point would map to a $k$-point of $Z$ lying over $t \in \mathbb{P}^{n}(k)-\mathbb{A}^{n}(k)$, contradicting the choice of $Z$.

Thus $\pi(Y(k))=U(k)$.

Remark 8.2. In the special case where $X$ is a regular projective curve and $U$ is an affine open subvariety of $X$, the reductions may be simplified greatly. Namely, using the Riemann-Roch theorem, construct a morphism $f: X \rightarrow \mathbb{P}^{1}$ such that $f^{-1}(\infty)=X-U$; now define $Y_{0}$ to make a cartesian diagram

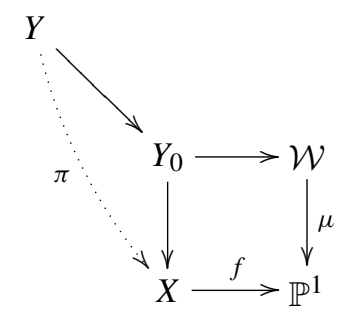

and let $Y$ be a resolution of singularities of $Y_{0}$. 


\section{Effectivity}

The construction of $Y$ in Theorem 1.3 as given is not effective, because it used Faltings' theorem. More specifically, in the proof of Lemma 7.1 we know that $C_{i}^{\prime}(k)$ is finite but might not know what it is, so when we reach the last paragraph of the proof, we might not know what the finite set $T$ is, and hence we have no algorithm for computing a good $c$, where good means that the images of $c$ and $T-\{0\}$ in $k^{\times} / k^{\times 2}$ do not meet.

Existence of an algorithm for Theorem 1.3 Let $F$ be the (finite) set of $t \in \mathbb{P}^{1}(k)$ such that $\mathcal{V}_{t}$ is not smooth. Suppose that instead of requiring that $c$ be good, we require only the effectively checkable condition that the images of $c$ and $F$ in $k^{\times} / k^{\times 2}$ do not meet. Then the proof of existence in Theorem 1.3 still yields a regular projective variety $Y_{c}$ and a morphism $\pi_{c}: Y_{c} \rightarrow X$, but it might not have the desired property $\pi_{c}\left(Y_{c}(k)\right)=U(k)$. Indeed, in the proof of Proposition 7.2, some of the Châtelet surfaces $\mathcal{W}_{t}$ other than $\mathcal{W}_{\infty}$ may be defined by a reducible degree- 4 polynomial and hence may violate the Hasse principle; thus the conclusion $\mu(\mathcal{W}(k))=\mathbb{A}^{1}(k)$ in Proposition 7.2 must be weakened to $\mu(\mathcal{W}(k)) \subseteq \mathbb{A}^{1}(k)$, and this eventually implies $\pi_{c}\left(Y_{c}(k)\right) \subseteq U(k)$.

On the other hand, an argument of Parshin (see [Szp85]) shows that Faltings' proof of the Mordell conjecture can be adapted to give an upper bound on the size of each set $C_{i}^{\prime}(k)$ in the proof of Lemma 7.1 Therefore we can compute a bound on \#T. Choose a finite subset $\Gamma \subseteq k^{\times}$whose image in $k^{\times} / k^{\times 2}$ is disjoint from the image of $F$ and has size greater than $\# T$. Then $\Gamma$ contains at least one $\operatorname{good} c$.

Let $Y=\bigsqcup_{c \in \Gamma} Y_{c}$, and define $\pi: Y \rightarrow X$ by $\left.\pi\right|_{Y_{c}}=\pi_{c}$. Then $\pi(Y(k))=$ $\bigcup_{c \in \Gamma} \pi_{c}\left(Y_{c}(k)\right)=U(k)$ since all terms in the union are subsets of $U(k)$ and some term equals $U(k)$.

\section{Algorithms for rational points}

Lemma 10.1. Any connected regular $k$-variety with a $k$-point is geometrically integral.

Proof. We give an argument that works over any ground field $k$.

Let $Y$ be the variety and let $y$ be the $k$-point. Then $Y$ is geometrically connected by [EGA IV.II] IV.4.5.14]. If $Y$ were reducible, there would be a point in the intersection of two irreducible components, and $Y$ would not be regular there. Therefore $Y$ is irreducible. Let $k_{s}$ be a separable closure of $k$, and let $Y_{s}=Y \times_{k} k_{s}$. Then $Y_{s}$ is regular [EGA IV.II IV.4.5.14] and connected, so by the same argument as above, $Y_{S}$ is irreducible. This suffices to prove that $Y$ is geometrically irreducible, by [EGA IV.II] IV.4.5.9].

Since $Y$ is regular, $Y$ is reduced. Combining this with the previous paragraph shows that $Y$ is integral. Since $Y$ is smooth at any regular $k$-point [EGA IV.IV] IV.17.15.1], there exists an open neighborhood $U$ of $y$ in $Y$ that is smooth, or equivalently, geometrically regular [EGA IV.IV], IV.17.15.2], which implies geometrically reduced. For an integral variety, being geometrically reduced depends only on the function field [EGA IV.II IV.4.6.1], so $Y$ is geometrically reduced too.

Combining the previous two paragraphs shows that $Y$ is geometrically integral [EGA IV.II, IV.4.6.2]. 
Proof of Theorem 1.1 $i$ ). Suppose that we want to know whether the $k$-variety $U$ has a $k$-point. By passing to a finite open cover, we may assume that $U$ is affine. Let $X$ be a projective closure of $U$. Construct $Y \rightarrow X$ as in Theorem 1.3 . Then $U$ has a $k$-point if and only if $Y$ has a $k$-point, so we reduce to the problem of deciding whether a regular projective variety $Y$ has a $k$-point. Connected components are computable, so we may assume that $Y$ is also connected. Check whether $Y$ is geometrically integral; if so, by assumption we can decide whether $Y$ has a $k$-point; if not, Lemma 10.1 implies that $Y$ has no $k$-point.

Proof of Theorem 1.1 $(\mathrm{ii})$. We want to compute \#X(k). Apply the algorithm of Theorem 1.1 (i) to $X$. If it says that $X$ has no $k$-point, we are done. Otherwise, search until a $k$-point $P$ on $X$ is found, and start over with the variety $X-\{P\}$. If $X(k)$ is finite, this algorithm will eventually terminate. (This kind of argument was also used in [Kim03].)

\section{Global function fields}

In this section, we investigate whether the proofs of the previous sections carry over to the case where $k$ is a global function field of characteristic $p>2$.

The main issues are:

(1) The two-part paper [CSS87a CSS87b], which is key to all our main results, works only over number fields. But it seems likely that the same proofs work, with at most minor modifications, over any global field of characteristic not 2.

(2) The proof of Theorem 1.3 uses resolution of singularities, which is not proved in positive characteristic. Moreover, the proof of Theorem 1.1 uses Theorem 1.3 so it also is in question. Without assuming resolution of singularities, one would obtain the weaker versions of Theorem 1.1 and 1.3 in which the word "regular" is removed from both.

There are a few other issues, but these can be circumvented, as we now discuss.

The proof of Proposition 5.1 works for any global function field $k$ of characteristic not 2: fix a place $\infty$ of $k$, let $\mathcal{O}_{k}$ be the ring of functions that are regular outside $\infty$, and replace the archimedean and 2 -adic conditions on $a$ and $b$ by the condition that $a$ and $b$ be squares in the completion $k_{\infty}$; then the proof proceeds as before.

The second paragraph of the proof of Lemma 7.1 encounters two problems in positive characteristic: first, it needs $v_{i}$ and $\beta$ to be separable, and second, to apply the function field analogue [Sam66] of Faltings' theorem it needs $C_{i}^{\prime}$ to be nonisotrivial. As for the first problem, if in Section 6 we choose $\tilde{P}_{\infty}(w, x)$ to be separable, then the same will be true of $\tilde{P}_{\infty}+v^{2} \tilde{P}_{0}$ over $k(v)$, and the same will be true of the $v_{i}$ in the application of Lemma 7.1, since the $v_{i}$ correspond to field extensions of $k(v)$ contained in the splitting field of $P_{\infty}+v^{2} \tilde{P}_{0}$ over $k(v)$; moreover, $\beta$ can be made separable simply by choosing $n$ not divisible by $p$. As for the second problem, the flexibility in the choice of coordinate used to define $\beta$ in the proof of Lemma 7.1 lets us arrange for $C_{i}^{\prime}$ to be nonisotrivial. Moreover, in this case, one can bound not only the number of $k$-points on each $C_{i}^{\prime}$, but also their height [Szp81, §8, Corollaire 2]. 
There is another thing that is better over global function fields $k$ than over number fields. Namely, by a proved extension of Hilbert's tenth problem to such $k$ [Phe91, Sh192, Vid94, Eis03], it is already known that there is no algorithm for deciding whether a $k$-variety has a $k$-point. Therefore, if $k$ is a global function field of characteristic not 2, and we assume that [CSS87a CSS87b] works over $k$, then there is no algorithm for deciding whether a projective geometrically integral $k$-variety has a $k$-point (and if we moreover assume resolution of singularities, we can add the adjective "regular" in this final statement).

Remark 11.1. Bianca Viray [Vir09] has proved an analogue of Proposition 5.1 for every global function field of characteristic 2 .

\section{Open questions}

(i) Can one generalize Remark 1.2 f) to show that to have algorithms as in (i) and (ii) of Theorem 1.1 for $n$-folds, it would suffice to be able to decide the existence of rational points on regular projective geometrically integral $(n+2)$-folds?

(ii) Is there a proof of Proposition 5.1 that does not require such explicit calculations?

(iii) Is the problem of deciding whether a smooth projective geometrically integral hypersurface over $k$ has a $k$-point also equivalent to the problem for arbitrary $k$-varieties?

Acknowledgments. I thank Jean-Louis Colliot-Thélène for several helpful comments, Brian Conrad for encouraging me to examine the function field analogue, Thomas Graber for a remark leading to Proposition 7.3. Bianca Viray for a correction to the proof of effectivity in Theorem 1.3 and J. Felipe Voloch and Olivier Wittenberg for suggesting some references. I thank the referee for many thoughtful suggestions towards making the exposition accessible to a broader audience.

This research was supported by NSF grant DMS-0301280.

\section{References}

[Co198] Colliot-Thélène, J.-L.: The Hasse principle in a pencil of algebraic varieties. In: Number Theory (Tiruchirapalli, 1996), Contemp. Math. 210, Amer. Math. Soc., Providence, RI, 19-39 (1998) Zbl 0910.14008 MR 1478483

[CCS80] Colliot-Thélène, J.-L., Coray, D., Sansuc, J.-J.: Descente et principe de Hasse pour certaines variétés rationnelles. J. Reine Angew. Math. 320, 150-191 (1980) Zbl 0434.14019 MR 592151

[CP00] Colliot-Thélène, J.-L., Poonen, B.: Algebraic families of nonzero elements of Shafarevich-Tate groups. J. Amer. Math. Soc. 13, 83-99 (2000) Zbl 0951.11022 MR 1697093

[CSS87a] Colliot-Thélène, J.-L., Sansuc, J.-J., Swinnerton-Dyer, P.: Intersections of two quadrics and Châtelet surfaces. I. J. Reine Angew. Math. 373, 37-107 (1987) Zbl 0622.14029 MR 870307

[CSS87b] Colliot-Thélène, J.-L., Sansuc, J.-J., Swinnerton-Dyer, P.: Intersections of two quadrics and Châtelet surfaces. II. J. Reine Angew. Math. 374, 72-168 (1987) Zbl 0622.14030 MR 876222 
[Eis03] Eisenträger, K.: Hilbert's tenth problem for algebraic function fields of characteristic 2. Pacific J. Math. 210, 261-281 (2003) Zbl 1057.11067 MR 1988534

[Fal83] Faltings, G.: Endlichkeitssätze für abelsche Varietäten über Zahlkörpern. Invent. Math. 73, 349-366 (1983); Erratum: Invent. Math. 75, 381 (1984) Zbl 0588.14026 MR 718935

[EGA IV.II] Grothendieck, A.: Éléments de géométrie algébrique. IV. Étude locale des schémas et des morphismes de schémas. II. Inst. Hautes Études Sci. Publ. Math. 24 (1965) Zbl 0135.39701 MR 0199181

[EGA IV.IV] Grothendieck, A.: Éléments de géométrie algébrique. IV. Étude locale des schémas et des morphismes de schémas IV. Inst. Hautes Études Sci. Publ. Math. 32 (1967) Zbl 0153.22301 MR 0238860

[Isk71] Iskovskikh, V. A.: A counterexample to the Hasse principle for systems of two quadratic forms in five variables. Mat. Zametki 10, 253-257 (1971) (in Russian) Zbl 0221.10028 MR 0286743

[Kim03] Kim, M.: Relating decision and search algorithms for rational points on curves of higher genus. Arch. Math. Logic 42, 563-568 (2003) Zbl 1038.11078|

[Lan54] Lang, S.: Some applications of the local uniformization theorem. Amer. J. Math. 76, 362-374 (1954) Zbl 0058.27201 MR 0062722

[Nis55] Nishimura, H.: Some remarks on rational points. Mem. Coll. Sci. Univ. Kyoto. Ser. A. Math. 29, 189-192 (1955) Zbl 0068.14802 MR 0095851

[Phe91] Pheidas, T.: Hilbert's tenth problem for fields of rational functions over finite fields. Invent. Math. 103, 1-8 (1991) Zbl 0696.12022 MR 1079837

[Sam66] Samuel, P.: Compléments à un article de Hans Grauert sur la conjecture de Mordell. Inst. Hautes Études Sci. Publ. Math. 29, 55-62 (1966) Zbl 0144.20102 MR 0204430

[Ser73] Serre, J.-P.: A Course in Arithmetic. Grad. Texts in Math. 7, Springer, New York (1973) Zbl 0256.12001 MR 0344216

[Ser79] Serre, J.-P.: Local Fields. Grad. Texts in Math. 67, Springer, New York (1979) Zbl 0423.12016 MR 554237

[Ser97] Serre, J.-P.: Lectures on the Mordell-Weil Theorem. Aspects Math. 3, Vieweg, Braunschweig (1997) Zbl 0863.14013 MR 1757192

[Shl92] Shlapentokh, A.: Hilbert's tenth problem for rings of algebraic functions in one variable over fields of constants of positive characteristic. Trans. Amer. Math. Soc. 333, 275-298 (1992) Zbl 0773.11075 MR 1091233

[Sko01] Skorobogatov, A.: Torsors and Rational Points. Cambridge Tracts in Math. 144, Cambridge Univ. Press, Cambridge (2001) Zbl 0972.14015 MR 1845760

[Smo91] Smoryński, C.: Logical Number Theory. I. Universitext, Springer, Berlin (1991) Zbl 0759.03002 MR 1106853

[Szp81] Szpiro, L.: Propriétés numériques du faisceau dualisant relatif. In: Séminaire sur les Pinceaux de Courbes de Genre au Moins Deux, Astérisque 86, 44-78 (1981) Zbl 0517.14006 MR 642675

[Szp85] Szpiro, L.: Un peu d'effectivité. In: Seminar on Arithmetic Bundles: the Mordell Conjecture (Paris, 1983/84), Astérisque 127, 275-287 (1985) MR 801928

[Vid94] Videla, C. R.: Hilbert's tenth problem for rational function fields in characteristic 2. Proc. Amer. Math. Soc. 120, 249-253 (1994) Zbl 0795.03015 MR 1159179

[Vir09] Viray, B.: Failure of the Hasse principle for Châtelet surfaces in characteristic 2. Preprint (2009) 